\title{
Accidente laboral. ACSOM una nueva orientación para gestión automática del riesgo
}

\author{
Work accidents. ACSOM a new orientation for automatic \\ risk management
}

\author{
A. I. García Felipe ${ }^{1}$, J.C. Conte ${ }^{1}$, E. Rubio ${ }^{1}$, A. Pérez Prados ${ }^{2}$
}

\section{RESUMEN}

Fundamento. Analizar la accidentalidad laboral en todo tipo de empresas, a través de los riesgos que la provocan, teniendo en cuenta que el objetivo final es establecer una clasificación de riesgos prioritarios. Esta información permite abordar las acciones preventivas oportunas sobre los riesgos más vulnerables.

Material y Métodos. Se aplica un conjunto de técnicas estadístico-matemáticas, basadas fundamentalmente en contrastes estadísticos, a los accidentes laborales acaecidos durante tres años, en un organismo de investigación y desarrollo español (I+D) con 8.780 trabajadores. Se utiliza como patrón de referencia en este estudio, para un análisis comparativo, el conjunto de accidentes en España durante un periodo de 11 años.

Resultados. Tras la aplicación de la nueva metodología, los dos primeros riesgos que requieren acciones preventivas prioritarias son interacción con substancias agresivas y accidentes con maquinaria móvil. Si únicamente se hubieran considerado los dos riesgos con frecuencias más elevadas, sin tener en cuenta la vulnerabilidad de cada uno de ellos, los riesgos prioritarios hubieran sido sobreesfuerzos y caídas al mismo nivel.

Conclusiones. El análisis proporciona una relación de los riesgos más vulnerables (puntos débiles), sobre los cuales actuar y de esta forma ser más efectivos a la hora de reducir la accidentalidad.

Palabras clave. Accidentes laborales. Riesgos laborales. Planificación de riesgos. Métodos estadísticos.

\begin{abstract}
Background. To analyse workplace accidents, by considering the risks that provoke such accidents, bearing in mind that the final aim is to establish a classification of priority risks. This information will make it possible to undertake the opportune preventive actions against the most vulnerable risks.
\end{abstract}

Material and Methods. A set of statistical-mathematical techniques, essentially based on statistical contrasts, was applied to work accidents taking place during a three year period in a Spanish Research and Development $(\mathrm{R}+\mathrm{D})$ organisation with 8,780 workers. The reference standard used for comparative analysis was the ensemble of accidents in Spain over an 11 year period.

Results. After application of the new methodology, the two risks requiring priority preventive actions are interaction with aggressive substances and accidents with moving machinery. If the two risks with the highest frequency had been considered alone, without taking into account the vulnerability of each, the priority risks would have been overexertion and falls at the same level.

Conclusions. The analysis provides a list of the most vulnerable risks accidents on which action should be taken and thus achieve greater effectiveness in establishing which actions should be considered opportune.

Key words. Work accidents. Work risk. Risk planning. Statistical methods.
1. Departamento de Microbiología, Medicina Preventiva y Salud Pública. Facultad de Medicina. Universidad de Zaragoza.

2. Departamento de Estadística e Investigación Operativa. Universidad Pública de Navarra.

Recepción: 11 de septiembre de 2008

Aceptación provisional: 5 de febrero de 2009

Aceptación definitiva: 25 de febrero de 2009

\section{Correspondencia:}

Ana Isabel García Felipe

Cátedra de Bioestadística

Facultad de Medicina. Universidad de Zaragoza

Domingo Miral s/n

50008 Zaragoza

Tfno. 976762727

Fax 976761704

E-mail: angarcia@unizar.es 


\section{INTRODUCCIÓN}

La necesidad de tratar la accidentalidad en las poblaciones laborales desde un punto de vista estadístico es un aspecto que se ha abordado clásicamente ${ }^{1-6}$.

También son clásicos los estudios matemáticos, considerando diferentes modelos de probabilidad. Aunque estos modelos se ajustan con el objetivo de prevenir la accidentalidad en problemas de salud, no se aplican a los accidentes laborales.

Otros enfoques metodológicos son los basados en la epidemiología y tampoco en este caso abundan las aplicaciones genéricas. En España el más general es el de Iglesia y col. ${ }^{7}$, quien ya sugiere la posibilidad de realizar estudios multivariantes, aspecto que se realiza puntualmente, también en problemas relacionados con la salud laboral.

La estadística multivariante permite reorientar el problema, darle una perspectiva global, y plantear una novedosa teoría del accidente ${ }^{8-10}$. Nuestra teoría integra el actual proceso de evaluación de riesgos y planificación preventiva ${ }^{11-13}$, así como otros aspectos del análisis clásico de accidentalidad con la investigación de accidentes ${ }^{14}$. A esta teoría la denominamos ACSOM, acrónimo de las palabras "ACcidents SOMa", que significa cuerpo de accidentes o comportamiento de los accidentes en una gran población.

Al conjunto de técnicas estadísticas ${ }^{15,16}$, variante de la minería de datos ${ }^{17,18}$, que se han utilizado para desarrollar la nueva teoría del accidente, se le denomina método Omega ${ }^{19,20}$.

Estas técnicas se han aplicado a todos los accidentes ocurridos en España, entre los años 1992-2002 $2^{21}$ con el fin de obtener un modelo de comportamiento que sirva de referencia. A este modelo, obtenido del conjunto total de datos de accidentes se le ha denominado ACSOM G, y es el que se toma como patrón general-poblacional. De forma análoga se procede para obtener los patrones de las distintas ramas de actividad, que se calcularán utilizando sólo los accidentes ocurridos en cada rama de actividad, y se denominan ACSOM S.

Si los datos proceden de una empresa, se denomina ProtoACSOM ${ }^{19,20}$ ya que es un ACSOM incompleto; en otras palabras, un
ACSOM aplicado a una parte reducida de la población; con muchos riesgos y lesiones con casuística muy baja o inexistente.

El método Omega permite todo tipo de comparaciones entre una parte de la población (ProtoACSOM) y el patrón general (puede ser el del país, ASCOM G, o el de una rama de actividad, ACSOM S). También se puede comparar el ACSOM S de cada rama de actividad con el ACSOM G.

$\mathrm{Al}$ estar basado en resultados obtenidos con técnicas estadísticas, el método indicado es científico y consecuentemente objetivo $^{9}$ y por ello se eliminan de su proceso todos los aspectos subjetivos, que las metodologías actuales necesitan adoptar, de forma complementaria, para alcanzar su desarrollo completo.

La aplicación de este método a los accidentes laborales proporciona a los técnicos de prevención una relación de riesgos detectados y ordenados según importancia, para que ellos consideren cuál debe ser la prevención a realizar. Pero también se puede obtener información análoga sobre las lesiones que se producen; en este caso, los resultados son de utilidad para los servicios médicos.

De esta manera puede llegar a ser una herramienta común de trabajo para todos los profesionales de la Prevención de Riesgos Laborales (PRL) tanto de los servicios médicos como técnicos. Sus resultados pueden ser la base de partida de la actividad preventiva médica y técnica, que hasta ahora no se consideraban de forma conjunta.

Las empresas a las que se ha aplicado este método son de distintas ramas de actividad y se ha verificado que el análisis frente al ACSOM G, es válido; no obstante, sería conveniente disponer de patrones de cada sector de actividad, dado que actividades muy específicas pueden generar accidentes más característicos.

En este artículo, se desarrolla el método y se aplica a un Organismo español de Investigación y Desarrollo, que se denomina $\mathrm{O}(\mathrm{I}+\mathrm{D})$. Se analizan los diferentes riesgos de la empresa $\mathrm{O}(\mathrm{I}+\mathrm{D})$ frente a los de ACSOM G, general de España, lo que conduce a la elaboración de una propuesta de intervención sobre los riesgos que se consideran más "vulnerables". 
Se entiende como vulnerables aquellos riesgos más susceptibles de reducir sus frecuencias, tras la implantación de las medidas correctoras oportunas. Es decir, son los riesgos considerados como puntos débiles, respecto al patrón de referencia. En otras palabras, se debería considerar que su frecuencia es inhabitual, por lo alta, en relación con la correspondiente del patrón, en esa empresa o actividad; esto es lo que le hace ser considerado como punto débil y por tanto como riesgo vulnerable, pues en él las acciones correctoras tendrán más eficacia.

Por el contrario, aquellos riesgos que aún pudiendo tener frecuencias elevadas, éstas estén por debajo de las del patrón, serán considerados como puntos fuertes o menos vulnerables, puesto que las medidas correctoras tendrán menos eficacia.

Para establecer la propuesta de intervención, se necesita tener ordenados los riesgos, de más vulnerable a menos vulnerable; esta clasificación se realiza con criterios estadísticos y a través de una secuencia de planificación se establece una ordenación de los mismos, o priorización.

Es importante resaltar que se ha desarrollado un programa informático, que permite obtener el informe final de priorización, por lo que facilita la tarea a los de- cisores, así como también facilita la tarea de los posteriores controles sobre los resultados obtenidos, una vez adoptadas las medidas preventivas.

En la última parte del artículo se inicia el estudio del comportamiento del ACSOM $\mathrm{S}$ de su rama de actividad, y se compara con el ProtoACSOM de la empresa y con el ACSOM G.

Por todo lo expuesto, el objetivo final del presente trabajo es tener información fiable para actuar en la reducción de la accidentalidad, reducción de la frecuencia de accidentes, mediante las acciones preventivas oportunas, sobre los riesgos más vulnerables (débiles).

\section{MATERIAL Y MÉTODOS}

Para el estudio se utilizan "registros bidimensionales" procedentes de los partes de accidentes en los que se define un Riesgo (R) y una Lesión (L). Al resumir los datos se obtiene una matriz $\mathrm{RxL}^{15,19,20}$ que funciona como un auténtico "Mapa de Riesgos" y, novedad, "Mapa de Lesiones".

Siguiendo la clasificación de la Organización Internacional del trabajo (OIT), se han considerado 19 tipos de riesgos y 19 lesiones (Tabla 1).

Tabla 1. Códigos de riesgos y lesiones.

\section{Códigos de Riesgos}
R1. Caídas a distinto nivel
R2. Caídas al mismo nivel
R3. Caídas de objetos por desplome
R4. Caídas de objetos por manipulación
R5. Caídas de objetos desprendidos
R6. Pisar sobre objetos
R7. Choques contra objetos inmóviles
R8. Choques contra objetos móviles
R9. Golpes, cortes por objetos o herramientas
R10. Proyección de fragmentos o partículas
R11. Atrapamiento por o entre objetos
R12. Accidentes con maquinaria móvil
R13. Sobreesfuerzos
R14. Exposición y/o contactos térmicos
R15. Contactos eléctricos
R16. Interacción con substancias agresivas
R17. Exposición a radiaciones
R18. Explosiones e incendios
R19. Accidentes causados por seres vivos

\section{Códigos de Lesiones}
L1. Fracturas
L2. Luxaciones
L3. Torceduras, esguinces y distensiones
L4. Lumbalgias
L5. Hernias discales
L6. Conmociones y traumatismos internos
L7. Amputaciones y pérdida del globo ocular
L8. Heridas abiertas. Otras heridas
L9. Traumatismos superficiales
L10. Contusiones o aplastamientos
L11. Cuerpos extraños en los ojos
L12. Conjuntivitis
L13. Quemaduras
L14. Envenenamientos e intoxicaciones
L15. Efectos ambientales
L16. Asfixias
L17. Efectos eléctricos
L18. Efectos por radiaciones
L19. Lesiones múltiples 
Si los datos provinieran de otros tipos de clasificación, mediante un sistema traductor se pueden convertir a códigos $\mathrm{OIT}^{18}$.

El patrón general, ACSOM G, está calculado, como ya se ha indicado, con los datos de todos los accidentes ocurridos en España durante 11 años (1992-2002) ${ }^{21}$. Se toma como patrón de comparación debido a que, por el considerable número de accidentes que incluye, el comportamiento que presenta se puede asumir como una situación media referencial. Nótese además que si se disminuye la accidentalidad laboral en cada empresa particular, se disminuirá la media a nivel nacional, consiguiéndose así rebajar tanto la media referencial como el número de accidentes. En el patrón general, ACSOM G, tras la aplicación de las diversas técnicas estadísticas se detectaron tres grupos, a los que se les asignó colores para una mejor identificación, sin ninguna connotación de gravedad ${ }^{10}$.

- Verde, se refiere a riesgos de mínima frecuencia y diversidad moderadamente significativa, denominados "del entorno".

- Amarillo, se refiere a riesgos de frecuencia media-alta, poca diversidad significativa y altísima relación con sus pares; es decir un riesgo muy asociado con una lesión y recíprocamente. Se les denomina "del individuo".

- Rojo, se refiere a riesgos de frecuencia media-alta y mayor diversidad significativa. Se les denomina "del individuo y entorno", por interacción entre ambos.

En la figura 1 se presenta un ejemplo de cada uno de ellos: R6 amarillo, R4 rojo y R15 verde.

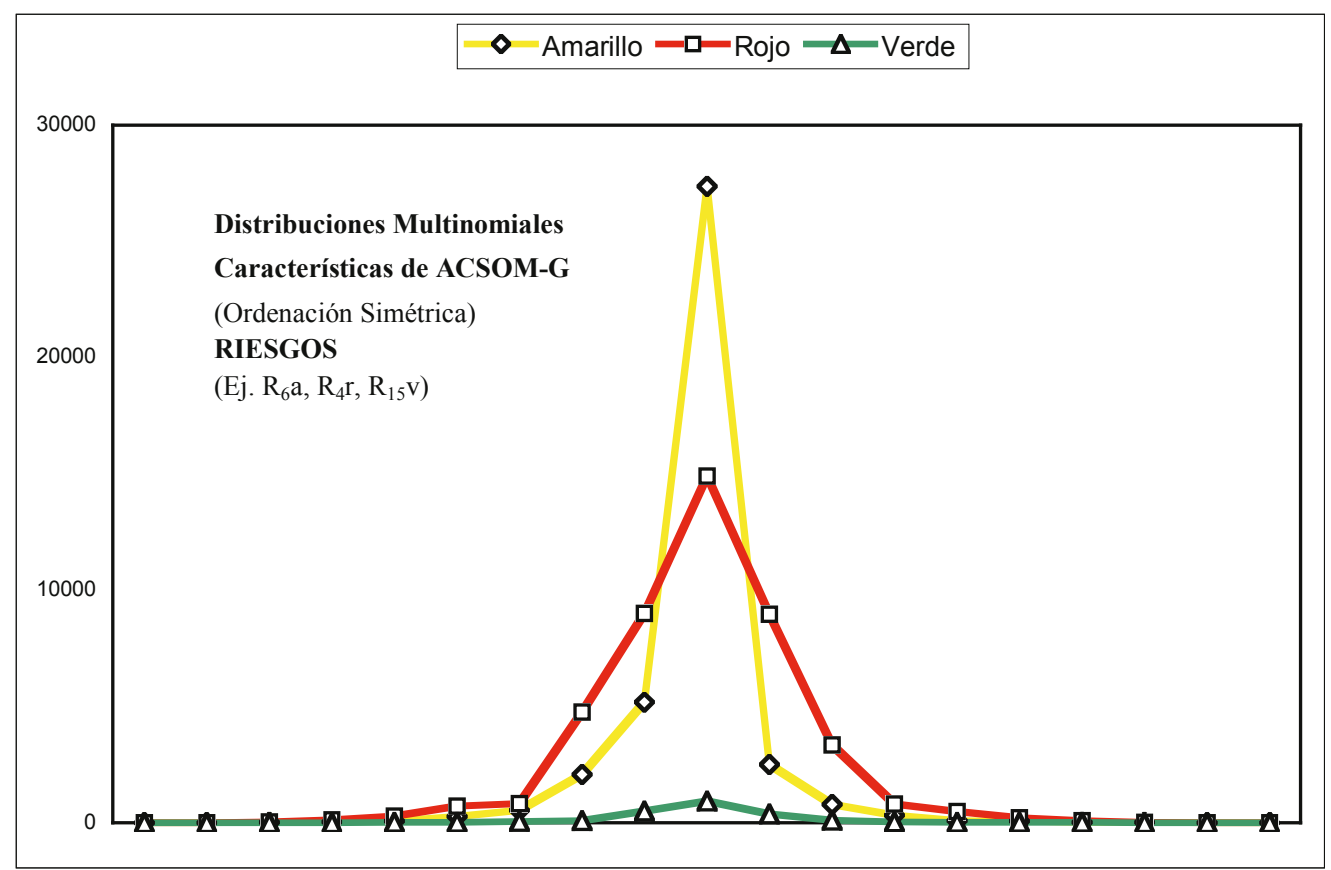

Figura 1. Perfil de tres riesgos de distinto grupo de color en ACSOM G.

\section{Material}

El organismo que se analiza, $\mathrm{O}(\mathrm{I}+\mathrm{D})$, tiene una población laboral media de 8.780 personas en el periodo de los tres años estudiados 2003, 2004 y 2005, durante el cual se produjeron 195 accidentes con algún día de baja laboral (Tabla 2). Se compara con el ACSOM G9 $\mathrm{G}^{9}$ con el ACSOM S de su rama de actividad calculado con los años $2000-2002^{21}$. 
Tabla 2. Matriz de riesgos y lesiones $\mathrm{O}(\mathrm{I}+\mathrm{D})$.

\begin{tabular}{|c|c|c|c|c|c|c|c|c|c|c|c|c|c|}
\hline \multirow[b]{2}{*}{ RIESGOS } & \multicolumn{13}{|c|}{ LESIONES } \\
\hline & L1 & $\mathbf{L} 2$ & L3 & L4 & L6 & L8 & L9 & L10 & L11 & L12 & L14 & L19 & Total \\
\hline R1 & 3 & & 7 & & 1 & & & 4 & & & & 1 & 16 \\
\hline R2 & 7 & 1 & 15 & & & & & 6 & & & & & 29 \\
\hline R3 & & & 1 & & 1 & & & 1 & & & & & 3 \\
\hline R5 & & & 1 & & & & 1 & 2 & & & & & 4 \\
\hline R7 & 1 & & 6 & 1 & & 1 & & & & & & 1 & 10 \\
\hline R8 & & & 2 & & & 1 & 4 & 2 & & & & & 9 \\
\hline R9 & 3 & & 1 & & & 14 & 3 & 3 & & & & & 24 \\
\hline R10 & & & & & & & & & 3 & & & & 3 \\
\hline R11 & 1 & & 2 & & & & 2 & & & & & & 5 \\
\hline R12 & 5 & & 6 & & & & 1 & 3 & & & & & 15 \\
\hline R13 & 1 & 2 & 28 & 23 & 3 & & 2 & 1 & & & & & 60 \\
\hline R14 & & & & & & & & & & & 2 & & 2 \\
\hline R16 & & & & & & & & & & 5 & 5 & & 10 \\
\hline R18 & & & & & & & & & & & 2 & & 2 \\
\hline R19 & 1 & & & & & 1 & 1 & & & & & & 3 \\
\hline Total & 22 & 3 & 69 & 24 & 5 & 17 & 14 & 22 & 3 & 5 & 9 & 2 & 195 \\
\hline
\end{tabular}

\section{Métodos estadísticos}

Dado que los datos se recogen en una tabla de contingencia, la metodología desarrollada analiza los distintos componentes de la tabla. En el cuerpo de la tabla, en otras publicaciones, se han aplicado diversas técnicas estadísticas multivariantes, para analizar la estructura latente de los riesgos/lesiones, del ProtoACSOM de la empresa estudiada y poderlos comparar con los correspondientes del patrón.

En este artículo la novedad es el análisis de uno de los marginales de la tabla, el correspondiente a los riesgos. Dado el alto contenido metodológico de este artículo y para una mejor comprensión de todo su desarrollo, está apoyado con una aplicación a la empresa mencionada, por lo que todas las referencias de este apartado, correspondientes a las figuras y tablas, exceptuando la tabla 3, están incluidas en el apartado de resultados.

Se realiza una comparación del polígono de frecuencias de los riesgos de $\mathrm{O}(\mathrm{I}+\mathrm{D})$ frente al del patrón referencial ACSOM G
(Fig. 2). Esta poligonal sigue el orden descendente de las frecuencias del patrón. Con la gráfica únicamente se puede tener una representación visual de la situación relativa de cada riesgo; pero, para cuantificar y priorizar los riesgos más vulnerables se necesita disponer de métodos analíticos.

Para realizar el análisis se establecieron tres secuencias: planificación Tipo I, Tipo II y Tipo III. Planificación Tipo I o "secuencia bruta" (Tabla 4), en la cual se presentan las posiciones que ocupan los diferentes riesgos de $\mathrm{O}(\mathrm{I}+\mathrm{D})$ en la línea superior y de ACSOM G en la inferior. Dichas posiciones quedan establecidas según el valor de las frecuencias relativas, desde el de mayor frecuencia que será el primero, al de menor que será el último; con la salvedad de que si la empresa estudiada tiene algún riesgo con casuística cero, desaparece de la secuencia; en el caso estudiado son 15 los riesgos que se manifestaron. Se obtienen con esta ordenación, los riesgos más frecuentes, pero no la potencial vulnerabilidad, o debilidad, de cada uno de ellos. 
Para ello, se valoran las diferencias con respecto al patrón; esto se realizará en los pasos siguientes. Así en la planificación Tipo II o "secuencia semidepurada" (Tabla 5 zona superior), se consideran las Translocaciones o diferencias de posiciones. Se compara la posición (asignando pseudo-rangos) que tiene cada riesgo en la empresa respecto a la que ocupa en el patrón (Tabla 4), y se calcula la diferencia entre pseudo-rangos.

Una vez ordenados los riesgos (Tabla 5), de mayor a menor valor diferencial, las diferencias positivas indican que ese riesgo está "peor", o más débil, en la empresa dado que aumenta la frecuencia respecto del patrón. A esta situación se le denomina "avanzada", puesto que se da un avance de posiciones en la empresa. Las diferencias negativas indicarán cuanto "mejor", o más fuertes, están que el patrón, a esta situación se le denomina "retrocedida", dado que se produce un retroceso de posiciones, pues la frecuencia en esos riesgos son menores que en el patrón. Si no se altera la posición, se dice que están "igual" y las frecuencias, en este caso, serán similares.

Seguidamente se realiza un análisis estadístico de contraste ${ }^{22}$ comparando la proporción de cada uno de los riesgos en la empresa frente a la del patrón mediante Chi-cuadrado y residuales ajustados de Haberman $^{23,24}$. Se valoran las desviaciones, mediante dichos residuales (Tabla 5 zona inferior), se consideran significativas las desviaciones con valores superiores a $+1,96$ o inferiores a - 1,96, asumiendo por tanto un error tipo I del 0,05 .

Por ello, si al realizar el contraste se obtienen cifras superiores a 1,96, se entiende que ese riesgo está "peor" que el patrón; en la tabla 4 se representa como "exceso"; si está por debajo de -1,96, estará "mejor" que el patrón y en la tabla 5 se representa como "defecto"; cuando sean valores no significativos se dice que están "igual", quedando ordenados los riesgos, de mayor a menor valor residual. Para aquellos riesgos en los que la frecuencia es baja, frecuencia esperada inferior a 5, se utiliza el residual de Freeman-Tukey ${ }^{25}$. Todos estos valores de Traslocación y Desviación están recogidos en la figura 3 .

Por último para establecer la ordenación o priorización de los riesgos se consideran las Translocaciones y Desviaciones en conjunto, según se valoren en primer lugar un criterio u otro, de este modo se obtienen dos posibles ordenaciones (Tabla 3a y Tabla $3 b)$. La información estaba proporcionada por la figura 3 (valoración de las Desviaciones y Translocaciones) y la tabla 5 que es la ordenación según los valores anteriores. En la tabla 3, se van situando los diferentes riesgos en las diferentes fases.

Tabla 3. Establecimientos de fases.

\begin{tabular}{|c|c|c|l|}
\hline Desviación & Translocación & Casiss & Riesgos de cada fase \\
\hline \hline \multirow{3}{*}{ Exceso } & $\mathrm{A}$ & 2 & $\mathrm{R}_{12}, \mathrm{R}_{16}$ \\
\cline { 2 - 4 } & $\mathrm{I}$ & 0 & \\
\cline { 2 - 4 } & $\mathrm{R}$ & 0 & \\
\hline \multirow{3}{*}{ Igual } & $\mathrm{A}$ & 5 & $\mathrm{R}_{2}, \mathrm{R}_{8}, \mathrm{R}_{s_{3}}, \mathrm{R}_{19}, \mathrm{R}_{7}$ \\
\hline & $\mathrm{I}$ & 3 & $\mathrm{R}_{13,}, \mathrm{R}_{18}, \mathrm{R}_{1}$ \\
\cline { 2 - 4 } & $\mathrm{R}$ & 2 & $\mathrm{R}_{3}, \mathrm{R}_{14}$ \\
\hline \multirow{3}{*}{ Defecto } & $\mathrm{A}$ & 0 & \\
\cline { 2 - 4 } & $\mathrm{I}$ & 0 & \\
\cline { 2 - 4 } & $\mathrm{R}$ & 3 & $\mathrm{R}_{11}, \mathrm{R}_{10}, \mathrm{R}_{9}$ \\
\hline
\end{tabular}

a) Desviación-Translocación

\begin{tabular}{|c|c|c|l|}
\hline Translocación & Desviación & Casos & Riesgos de cada fase \\
\hline \multirow{3}{*}{ Avanzada } & $\mathrm{E}$ & 2 & $\mathrm{R}_{15}, \mathrm{R}_{12}$ \\
\cline { 2 - 4 } & $\mathrm{I}$ & 5 & $\mathrm{R}_{5}, \mathrm{R}_{2}, \mathrm{R}_{8}, \mathrm{R}_{19}, \mathrm{R}_{7}$ \\
\cline { 2 - 4 } & $\mathrm{D}$ & 0 & \\
\hline \multirow{3}{*}{ Igual } & $\mathrm{F}$ & 0 & \\
& $\mathrm{I}$ & 3 & $\mathrm{R}_{13}, \mathrm{R}_{18}, \mathrm{R}_{1}$ \\
\cline { 2 - 4 } & $\mathrm{D}$ & 0 & \\
\hline \multirow{3}{*}{ Retrocedida } & $\mathrm{L}$ & 0 & \\
\cline { 2 - 4 } & $\mathrm{I}$ & 2 & $\mathrm{R}_{33} \mathrm{R}_{14}$ \\
\cline { 2 - 4 } & $\mathrm{D}$ & 3 & $\mathrm{R}_{9,} \mathrm{R}_{11}, \mathrm{R}_{10}$ \\
\hline
\end{tabular}

b) Translocación-Desviación
Como consecuencia, en la subtabla izquierda “3a) Desviación- Translocación”, el riesgo con el valor más elevado en "Exceso" de Desviación y el más elevado en "Avanzada" de Translocación, será el que esté en peor situación, es decir, el riesgo más débil o vulnerable en la empresa y será, de entre los que tienen frecuencia por encima del patrón, el de mayor diferencia significativa. Por el contrario, estará en la mejor situación el que tenga el valor más bajo en "Defecto" y en "Retrocedida" res- 
pectivamente, es decir, el riesgo más fuerte será, de entre los que tienen frecuencia por debajo del patrón, el de mayor diferencia significativa. De manera análoga se situarán en la subtabla " $5 b$ ) Translocación-Desviación”. En este caso se valorará primero Translocación.

De esta forma quedan establecidos los riesgos por fases de actuación. La primera fase será la de actuación prioritaria, quedando recogidos en la última fase los riesgos que están en la mejor situación en la empresa.

Con este procedimiento se resuelve la Planificación Tipo III. Son "secuencias depuradas" ya que incluyen las dos formas de optimizar, se resuelve por ambas ordenaciones de criterios, para mostrar las diferencias que presentan (Tabla 6).

Posteriormente se presentan en un mismo gráfico (Fig. 4), el polígono de frecuencias del ACSOM S, de su rama de actividad, se realiza un análisis muy superficial dado que es una aproximación, ACSOM S simplificado con solo dos años de accidentes; junto con el polígono de frecuencias de ACSOM G y el del ProtoACSOM O(I+D), para comprobar las diferencias que se pueden presentar, si se utilizase como patrón el de su rama de actividad en lugar de utilizar el patrón general.

\section{RESULTADOS}

\section{Contraste entre los Riesgos de $\mathrm{O}(\mathrm{I}+\mathrm{D})$ y ACSOM G}

En la figura 2, se presenta la gráfica poligonal que compara el ProtoACSOM O(I+D) con ACSOM G, se muestra la situación de cada riesgo en la empresa estudiada respecto de su patrón de referencia.

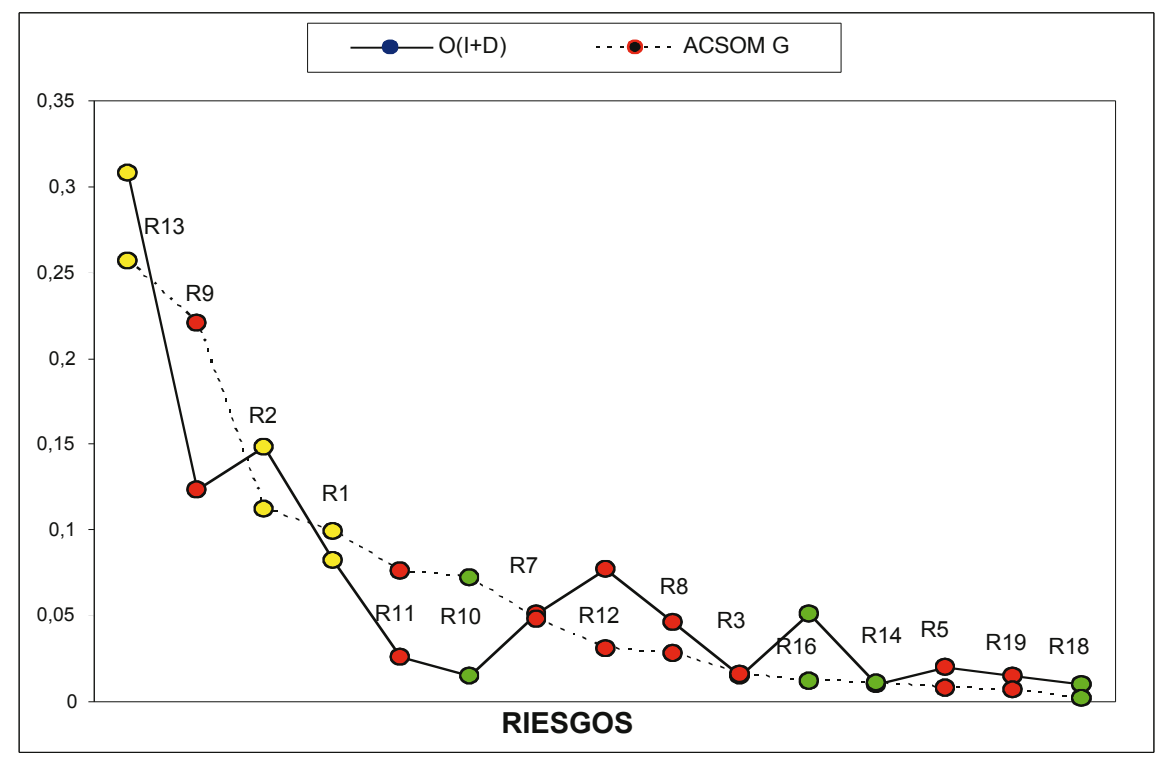

Figura 2. Poligonales de los Riesgos O(I+D) vs ACSOM G.

En la figura 3, se presentan las valoraciones de las Translocaciones y los valores de Desviaciones, mediante dos barras adosadas para cada riesgo, al comparar el ProtoACSOM de O(I+D) con ACSOM G, estos resultados presentan los riesgos que se encuentran en mejor o peor situación. 


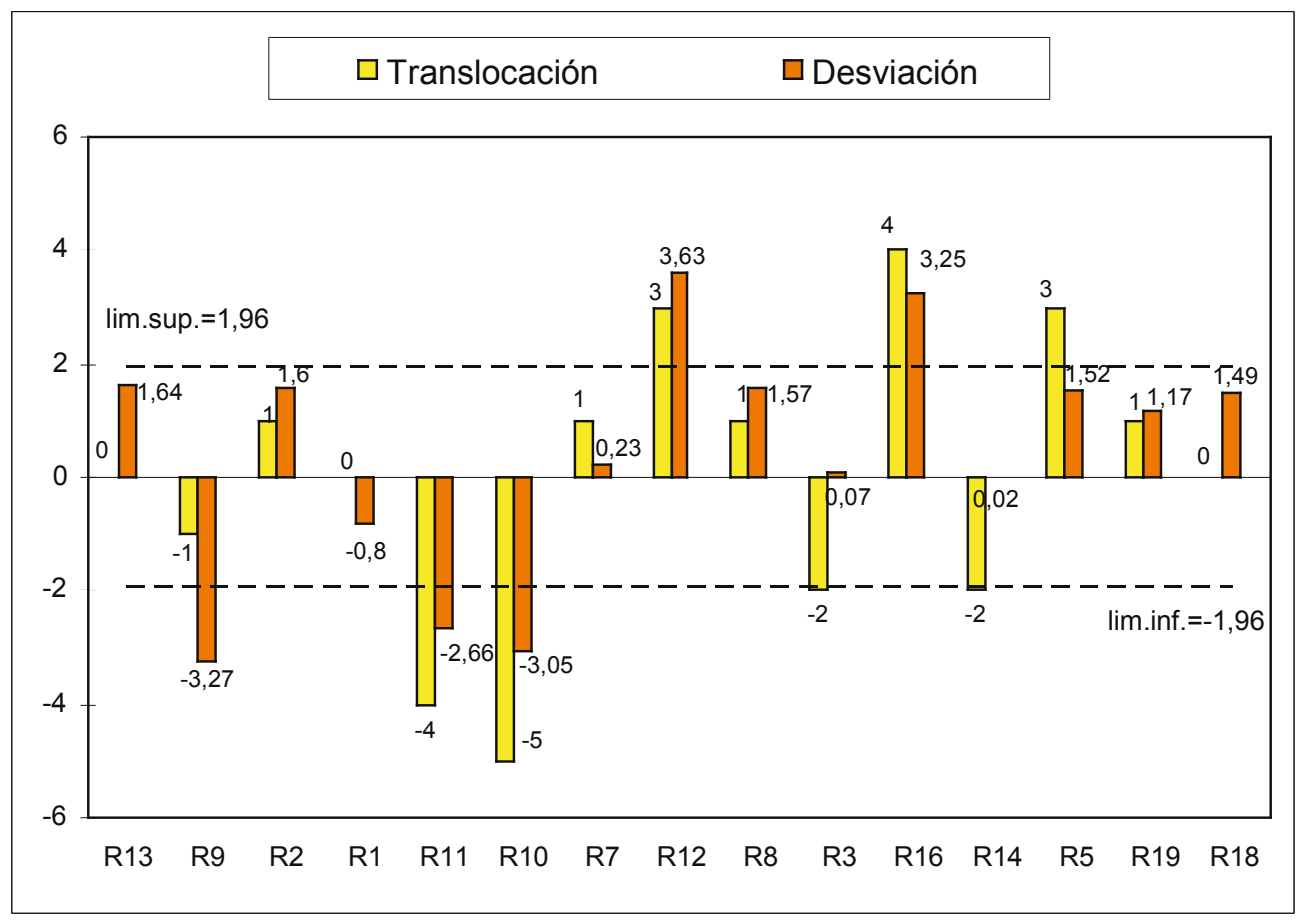

Figura 3. Translocaciones y Desviaciones O(I+D) vs ACSOM G.

\section{Planificación de riesgos prioritarios en $\mathbf{O}(\mathrm{I}+\mathrm{D})$}

En la tabla 4 quedan situados los riesgos por orden descendente de las frecuen- cias, según el valor que toma en la empresa $\mathrm{O}(\mathrm{I}+\mathrm{D})$ y en el patrón. Se puede observar como existen claras diferencias en ambos órdenes.

Tabla 4. Planificación Tipo I (secuencia bruta).

\begin{tabular}{|l|l|l|l|l|l|l|l|l|l|l|l|l|l|l|}
\hline \multicolumn{110}{|c|}{ Posición O(I+D) } \\
\hline $\mathrm{R}_{13}$ & $\mathrm{R}_{2}$ & $\mathrm{R}_{9}$ & $\mathrm{R}_{1}$ & $\mathrm{R}_{12}$ & $\mathrm{R}_{7}$ & $\mathrm{R}_{16}$ & $\mathrm{R}_{8}$ & $\mathrm{R}_{11}$ & $\mathrm{R}_{5}$ & $\mathrm{R}_{10}$ & $\mathrm{R}_{3}$ & $\mathrm{R}_{19}$ & $\mathrm{R}_{14}$ & $\mathrm{R}_{18}$ \\
\hline \multicolumn{10}{|c|}{ Posición (ACSOM G) } \\
\hline $\mathrm{R}_{13}$ & $\mathrm{R}_{9}$ & $\mathrm{R}_{2}$ & $\mathrm{R}_{1}$ & $\mathrm{R}_{11}$ & $\mathrm{R}_{10}$ & $\mathrm{R}_{7}$ & $\mathrm{R}_{12}$ & $\mathrm{R}_{8}$ & $\mathrm{R}_{3}$ & $\mathrm{R}_{16}$ & $\mathrm{R}_{14}$ & $\mathrm{R}_{5}$ & $\mathrm{R}_{19}$ & $\mathrm{R}_{18}$ \\
\hline
\end{tabular}

Profundizando en el análisis se continúa con planificación tipo II (Tabla 5), en primer lugar la clasificación por valores de Translocaciones y, en segundo término, por valores de Desviación, según los valores presentados en la figura 3 . 
Tabla 5. Planificación de Tipo-II (secuencia semidepurada, criterio Translocación y Desviación).

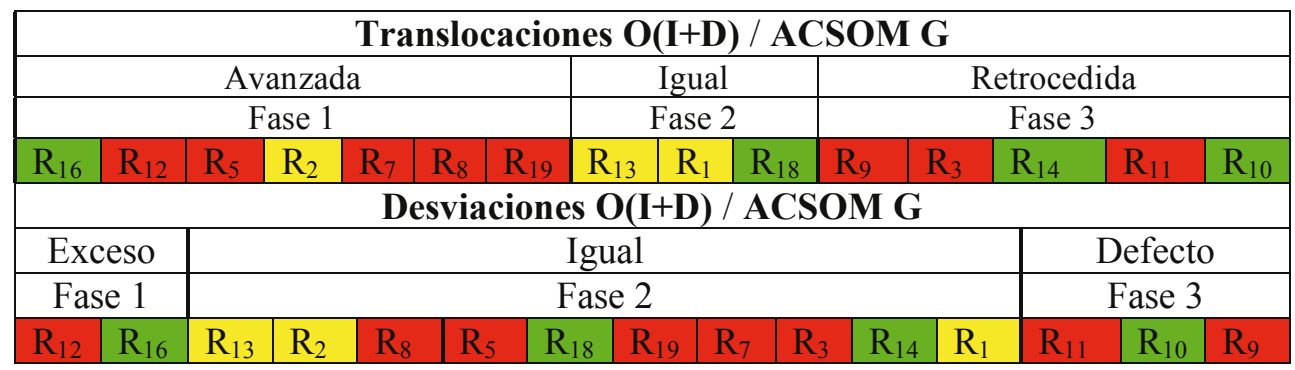

En la última fase de planificación (Tabla 6), se tiene el informe final por fases de actuación. La Planificación de la priorización de los riesgos de $\mathrm{O}(\mathrm{I}+\mathrm{D})$, cuando se consideran los dos criterios indicados.
Este procedimiento proporciona dos posibles soluciones, según se considere en primer lugar las Translocaciones o Desviaciones, se obtienen dos secuencias de resultados.

Tabla 6. Planificación Tipo-III (secuencia depurada, priorizando Translocaciones o Desviaciones).

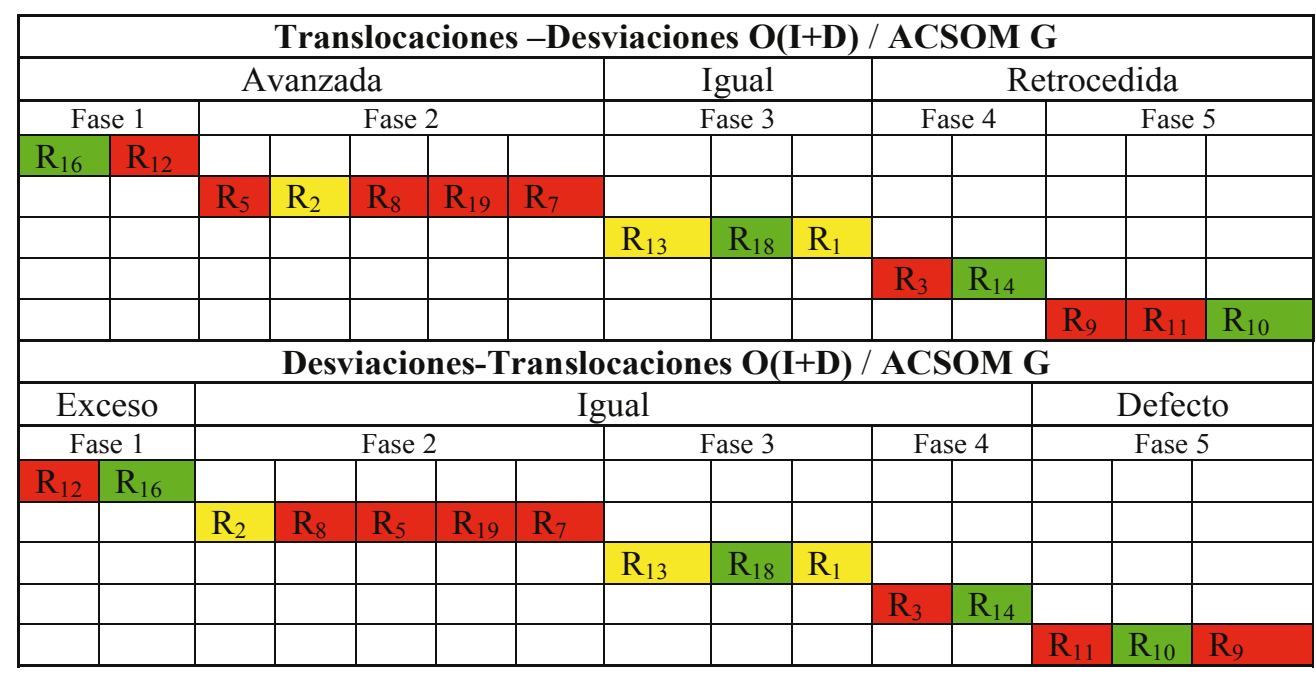

\section{Comparación de O(I+D) con el ACSOM S simplificado de su rama de actividad y ACSOM G}

Como última parte del análisis (Fig. 4), se presenta la comparación con un ACSOM
S simplificado de la actividad que corresponde a la empresa $\mathrm{O}(\mathrm{I}+\mathrm{D})$.

Para ello, en el mismo gráfico se incluyen las poligonales de $\mathrm{O}(\mathrm{I}+\mathrm{D}), \mathrm{ACSOM} \mathrm{G} \mathrm{y}$ el ACSOM S simplificado de su rama de actividad. Permite de este modo apreciar las diferencias entre las tres poligonales. 


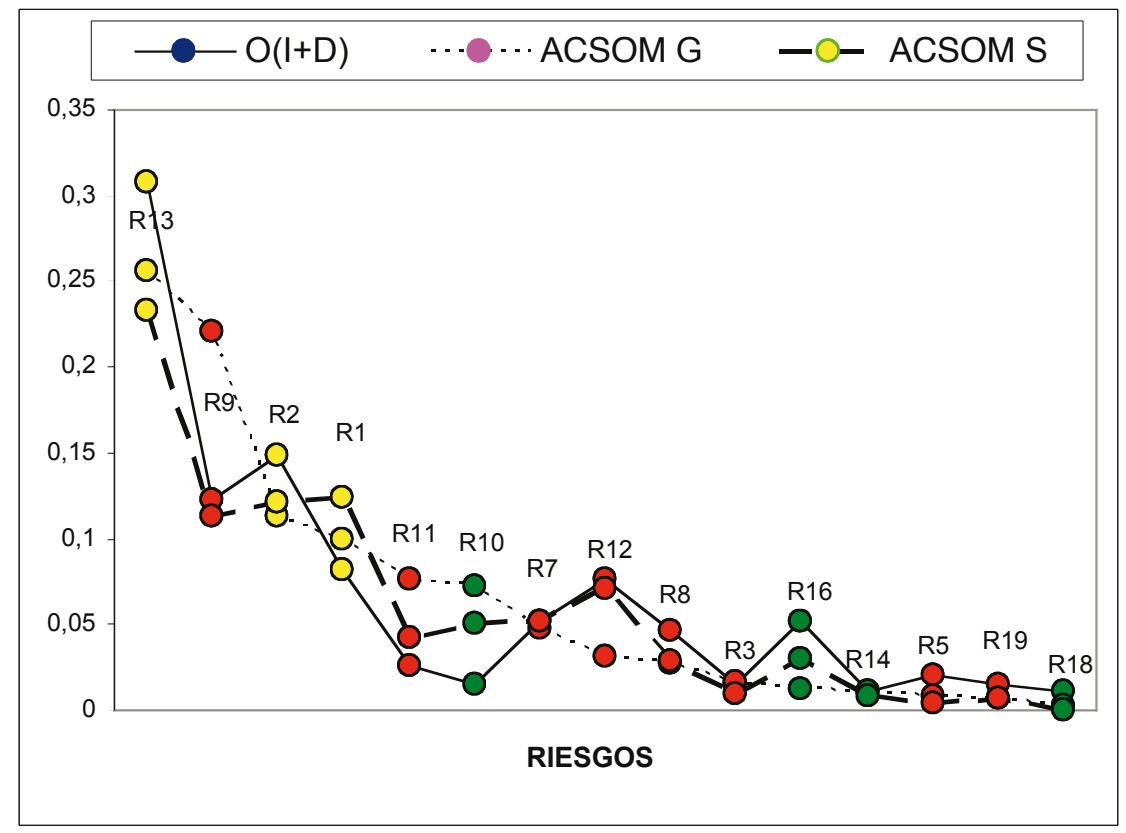

Figura 4. Poligonales de los Riesgos. ProtoACSOM O(I+D), ACSOM S simplificado O(I+D) y ACSOM G.

\section{DISCUSIÓN}

En la figura 2, se observa cómo se sitúan cada uno de los riesgos y su importancia relativa, por encima de los valores del patrón R13, R2, R12, R16 como más relevantes y por debajo R9, R1, R11, R10; pero, para establecer un orden de vulnerabilidad, se necesita completar la información mediante una cuantificación de las diferencias.

En la figura 3 se observa que hay riesgos con Translocaciones superiores a $0 \mathrm{y}$ Desviaciones que superan el valor $+1,96$; estos serán los riesgos prioritarios. Tomando únicamente los más relevantes R12 y R16 son los que tienen la peor situación, son puntos débiles en la empresa O(I+D), y R11 y R10 (el mejor), son puntos fuertes en la empresa $\mathrm{O}(\mathrm{I}+\mathrm{D})$

Hasta llegar al informe final o propuesta de actuación prioritaria es preciso recorrer las distintas secuencias de planificación. En primer lugar, en la tabla 4, los riesgos más frecuentes son R13, R2, R9 y R1, coinciden con las cuatro primeras de ACSOM G; aunque hay una alteración en el orden de la segunda y la tercera.
Si se pretende establecer una política preventiva, el hecho de actuar en primer lugar sobre estos riesgos porque presentan mayor frecuencia, sin tener en cuenta la situación frente el patrón, puede resultar ineficaz.

Si se observa la figura 2, R13 y R2 tienen una frecuencia más elevada que el patrón, por el contrario R9 y R1 la tienen más baja, es decir en principio se deberían elegir R13 y R2 y no considerar inicialmente R9 y R1.

Pero, para hacerlo de forma objetiva, se necesita conocer cuáles son los riesgos más vulnerables; la teoría ACSOM considera que serán aquellos que presenten frecuencias superiores a las del patrón y dentro de ellas las que mayor importancia relativa tengan. Para ello se realiza, en primer lugar, la clasificación por Translocaciones, planificación tipo II (Tabla 5). Hay que recordar aquí que se ha considerado "avanzada" o "retrocedida" cualquier puntuación positiva o negativa respectivamente. El orden de los cuatro primeros riesgos más débiles o vulnerables, es: R16, R12, R5 y R2. 
Esto significa que con este criterio estos cuatro riesgos son los prioritarios, dado que R16 respecto al patrón es el que más ha aumentado su frecuencia; pasa de ocupar el puesto (Tabla 4), $11^{\circ}$ en ACSOM $\mathrm{G}$ al $7^{\circ}$ en $\mathrm{O}(\mathrm{I}+\mathrm{D})$, en cuanto a R12 pasa del $8^{\circ}$ al $5^{\circ}$ y así sucesivamente, tomando por tanto valores de Translocación (Fig. 3, de 4 y 3 respectivamente).

Para completar esta información se necesita el segundo criterio el de Desviación (Tabla 5), como se ordenan de mayor a menor los valores, en la tabla quedan situados los riesgos desde una peor a una mejor situación. Seleccionando los cuatro "peores" o más débiles, el orden es, R12, R16, R13 y R2. Quedando como "mejores", más fuertes, R11, R10 y R9.

Por tanto R12 y R16 que habían presentado las Translocaciones más elevadas, coinciden también con las Desviaciones más elevadas, 3,63 y 3,25 (Fig. 3), aunque cambiando el orden; también se presentan algunas variaciones de orden en los siguientes riesgos. Ello conduce a la siguiente fase, que consiste en considerar en conjunto Translocaciones y Desviaciones.

En tabla 6, los dos riesgos de actuación prioritaria en ambas secuencias aunque con orden distinto son: R16, R12, por eso se proponen de atención prioritaria en una primera fase. En la fase 2, coinciden todos los riesgos, pero no ocupan los mismos puestos. Así, si se consideran en primer lugar translocaciones, los tres primeros de esta fase por orden son R5, R2 y R8. Si se consideran en primer lugar desviaciones, el primer riesgo de esta fase 2 es R2, seguido de R8. Se demuestra con ello que las diferencias son mínimas.

Es de señalar que sin aplicar esta metodología, exceptuando R2, estos riesgos eran secundarios; R16 (Tabla 4), posición O(I+D) ocupaba el $7^{\circ}$ lugar, R12 el 5ㅜㅇ R5 el 10ㅜ; R8 el $8^{\circ}$ etc... No son por tanto los que presentan las frecuencias más elevadas, pero con respecto de la media de accidentes en España, representados por ACSOM G, si que están peor y de forma significativa.

Estos riesgos seleccionados como más vulnerables, en muchos casos coinciden con los que provocan los accidentes más graves como pueden ser R12 y R16, aunque este estudio no analiza esta característica de los riesgos.
No obstante, es importante tener en cuenta todos los resultados del proceso de planificación, puesto que todos aportan aspectos importantes que son necesarios conocer y valorar, junto con la posible gravedad de las consecuencias de estos riesgos. También se deberían considerar las políticas a nivel Nacional o Sectorial, para consensuar una correcta toma de decisiones, una vez que los técnicos dispongan de una relación de actuaciones prioritarias.

En la última parte del análisis se ha realizado un estudio preliminar sobre el ACSOM $\mathrm{S}$ de la rama de actividad de la empresa, puesto que el estudio sería más preciso si se crearan los patrones de las diferentes ramas de actividad, ya que en este caso los puntos débiles y fuertes estarían más ajustados.

En la figura 4, se observa que aún siendo solo un ACSOM S con datos de solo dos años, ya se comporta de manera diferenciada de ACSOM G; a simple vista se puede apreciar las diferencias mas relevantes se aproximan a $\mathrm{O}(\mathrm{I}+\mathrm{D}) \mathrm{R} 9, \mathrm{R} 11, \mathrm{R} 10, \mathrm{R} 12, \mathrm{R} 16$ y se alejan R13, R1 y R5.

Por tanto, si este ACSOM fuera el verdadero ACSOM de su rama de actividad, esto conduciría a modificar las órdenes de prioridad analizadas anteriormente, puesto que, al utilizarlo como patrón hubiera empeorado su situación respecto a la que presentaban anteriormente, R13, R9, R1, R11, R10, R5 y hubiera mejorado R12 y R16 esto se ha obtenido realizando únicamente un análisis gráfico de la nueva situación.

Como conclusión, se ha mostrado la utilidad de la teoría ACSOM como método objetivo en el análisis de riesgos de un organismo I+D al compararlo con el ACSOM G de España.

El análisis de los riesgos marginales proporciona una relación de los riesgos más vulnerables, puntos débiles, sobre los cuales actuar.

Según el proceso de planificación, los riesgos que requieren una intervención preventiva prioritaria en $\mathrm{O}(\mathrm{I}+\mathrm{D})$, ordenados por vulnerabilidad, en una primera fase, son Interacción con substancias agresivas (R16) y Accidentes con maquinaria móvil (R12).

En una segunda fase, caídas de objetos desprendidos (R5), caídas al mismo nivel (R2), choques con objetos móviles e inmó- 
viles (R8, R7), accidentes causados por seres vivos (R19).

Si únicamente se hubieran considerado los de máxima frecuencia, solo caídas al mismo nivel (R2), se hubiera tenido en cuenta como actuación preventiva prioritaria.

Se ha visto, gráficamente, que existen diferencias entre el ACSOM S simplificado de su rama y el ACSOM G. Si se tuviesen los patrones de las ramas de Actividad del Sector, se experimentaría una mejora en la precisión de la planificación de los riesgos en las empresas. Esto sugiere la necesidad de creación de los ACSOM S reales.

Finalmente es conveniente señalar aquí que, aunque este estudio no analiza ni la gravedad de los accidentes ni sus repercusiones económicas, son dos líneas interesantes para abordar en investigaciones posteriores, en cada una de las ramas de actividad.

\section{BIBLIOGRAFÍA}

1. Organización Internacional del Trabajo. X Conferencia Internacional de Estadísticos del Trabajo. OIT. Ginebra 1962.

2. Organización Internacional del Trabajo. XIII Conferencia Internacional de Estadísticos del Trabajo. OIT. Ginebra 1982.

3. De las Heras E. Metodología para la realización de investigaciones y análisis de accidentes/ incidentes. Asociación para la Prevención de Accidentes CAPA. 1995.

4. AlCÁntARA A. Estadística en relaciones laborales. Hespérides. Salamanca. España. 1999.

5. Bestratén M, Gil A, PiQué A. La gestión integral de los accidentes de trabajo (I): tratamiento documental e investigación de accidentes NTP-592. INSHT 2003.

6. Bestratén M, Gil A, Piqué A. La gestión integral de los accidentes de trabajo (II): Control Estadístico NTP-593. INSHT 2003b.

7. De la iglesia Huerta A, Solé Gómez MD, Marqués Marqués F, Pérez Nicolás J. Epidemiología laboral. INSHT 1992.

8. Conte JC, Rubio E, Domínguez AI, García AI. Análisis de la problemática preventiva para el control de la accidentalidad laboral. Arch Fac Med Zar Univ Zaragoza 2004; 44: 113-118.

9. Conte JC, García AI, Rubio B. Teoría del accidente en poblaciones. Cuad Bioestad Apl Inform 2007a; 17: 26-38.
10. Conte JC, Marcos G, García AI, Rubio E. Análisis del problema empírico de identificación del riesgo. Cuad Bioestad Apl Inform 2007b; 17: 12-25.

11. BiRd FE (JR), Gennain GL. Damage Control. American Management Association. Nueva York 1966.

12. FInE W. Mathematical Evaluations for Controlling Hazards. Academic Press. Col. Selected Reading in Safety. Georgia. 1973.

13. BIRD FE (JR), GenNAIN GL. Liderazgo práctico en el control de pérdidas. international Loss Control Institute, USA 1990.

14. TENEO. Gestión de la seguridad industrial. Grupo Teneo. 1995.

15. Conte JC, Rubio E. Estudio de la relación riesgo-lesión mediante análisis factorial. R Acad Cienc Exactas Fís Quím Nat Zaragoza 2005; 60: 65-85.

16. Conte JC, Cano F, García AI, Molina A, Rubio E. Interpretación de las relaciones intragrupales de riesgos y de lesiones mediante análisis cluster jerárquico. Revista de matemáticas teoría y aplicaciones 2008; 15: 175-184.

17. REAL JE. Escalamiento multidimensional. La Muralla. S.A. Madrid. 2001.

18. Rubio E, Conte JC, García AI. Minería de datos, Salud Laboral y Sistemas Inteligentes. Cuad Bioestad Apl Inform 2007a; 17: 2-11.

19. Conte JC, Rubio E, García AI, Domínguez AI, $\mathrm{OMEGA}^{\circledR}$. Metodología para la previsión del accidente en poblaciones laborales. Aplicación a Microempresas. Med Segur Trab 2006a; 52 (203): 27-37.

20. Conte JC, Rubio E, García AI, Domínguez AI. Método OMEGA ${ }^{\circledR}$ : Aplicación a pequeñas y grandes empresas. Med Segur Trab 2006b; 52 (203): 39-49.

21. Ministerio de Trabajo y Asuntos Sociales. Estadística de accidentes de Trabajo. Madrid. España. 1992-2002.

22. Rubio E, García AI. Estadística y Medicina del Trabajo. En: Ergonomía, 20 preguntas básicas para aplicar la ergonomía a la empresa. Mapfre. Madrid. 2001; 211-286.

23. García AI, Alvarez JM, Alcalde V, Rubio B, BasCUAS J. Monitorización epidemiológica en ciencias de la salud mediante el residual de incidentalidad estandarizado (RIS). MapfreSeguridad 2004; 93: 33-42.

24. Ruiz L, Martín FJ, Montero JM, Uriz-Tomé P. Análisis estadístico de encuestas: datos cualitativos. AC Editorial. Madrid. España 1995.

25. SALIFU M. Accident prediction models for unsignalised urban junctions in Ghana. IATSS Research 2004; 28: 68-81. 